\title{
Twisted Surfaces in the Pseudo-Galilean Space
}

\author{
Ahmet Kazan ${ }^{1}$, H.Bayram Karadag ${ }^{2}$ \\ ${ }^{1}$ Department of Computer Technologies, Surgu Vocational School of Higher Education, Inonu University, Malatya, Turkey \\ ${ }^{2}$ Department of Mathematics, Faculty of Arts and Sciences, Inonu University, Malatya, Turkey
}

Received: 3 August 2016, Accepted: 26 February 2017

Published online: 25 October 2017.

\begin{abstract}
In this paper, we construct the twisted surfaces according to the supporting plane and type of rotations in pseudo-Galilean space $G_{3}^{1}$. Also, we find the Gaussian curvatures and mean curvatures of the different types of these twisted surfaces and draw some figures for these twisted surfaces.
\end{abstract}

Keywords: Twisted surface, Pseudo-Galilean space, Pseudo-Euclidean rotation, Isotropic rotation.

\section{Introduction}

Many types of surfaces, for example surfaces of revolution, ruled surfaces, helical surfaces and etc., have been studied in Euclidean and pseudo-Euclidean spaces recently ([1],[4],[5],[11]-[13] and etc). Also in [3], Cervone has investigated tight polyhedral immersion of the twisted surface of Euler characteristic - 3. After this, Goemans and Woestyne have examined twisted surfaces which are generated by synchronized rotations of a planar curve in its supporting plane and of this supporting plane about some axis in Euclidean 3-space and Minkowski 3-space ([8]-[10]). The resulting surfaces can be seen as generalizations of surfaces of revolution. In those articles, they have obtained classifications of flat, constant Gaussian curvature and constant mean curvature (regular) twisted surfaces. They also have showed that, there exists no minimal (regular) twisted surface in Euclidean or in Minkowski 3-space when excluding the surfaces of revolution.

Here, our aim to investigate twisted surfaces in pseudo-Galilean space $G_{3}^{1}$ which is one of the model of Cayley-Klein geometries. About the geometries of curves and surfaces in Galilean and pseudo-Galilean spaces, there are lots of studies in the literature ([2],[16],[17] and etc).

In this study, we will construct the twisted surfaces according to the supporting plane and type of rotations in pseudo-Galilean space $G_{3}^{1}$. Also, we will find the Gaussian curvatures and mean curvatures of the different types of these twisted surfaces and draw some figures for these twisted surfaces with the aid of Wolfram Mathematica.

\section{Preliminaries}

Here, firstly we will recall some notations about pseudo-Galilean space.

The pseudo-Galilean space $G_{3}^{1}$ is a Cayley-Klein space with absolute figure consisting of the ordered triple $\{w, f, I\}$, where $w$ is the ideal (absolute) plane in the three dimensional real projective space $R P 3, f$ the line (the absolute line) in $w$ and $I$ the fixed hyperbolic involution of points of $f$. 
In affine coordinates defined by $\left(x_{0}: x_{1}: x_{2}: x_{3}\right)=(1: x: y: z)$, the distance between the points $P_{i}=\left(x_{i}, y_{i}, z_{i}\right)(i=1,2)$ is defined by [7]

$$
d\left(P_{1}, P_{2}\right)= \begin{cases}\left|x_{2}-x_{1}\right|, & \text { if } x_{1} \neq x_{2}, \\ \sqrt{\left|\left(y_{2}-y_{1}\right)^{2}-\left(z_{2}-z_{1}\right)^{2}\right|}, & \text { if } x_{1}=x_{2} .\end{cases}
$$

In affine coordinates, the group motions of $G_{3}^{1}$ is a six-parameter group given by

$$
\begin{aligned}
& \bar{x}=a+x, \\
& \bar{y}=b+c x+y \cosh \varphi+z \sinh \varphi, \\
& \bar{z}=d+e x+y \sinh \varphi+z \cosh \varphi .
\end{aligned}
$$

The pseudo-Galilean scalar product can be written as

$$
\left\langle v_{1}, v_{2}\right\rangle= \begin{cases}x_{1} x_{2}, & \text { if } x_{1} \neq 0 \vee x_{2} \neq 0 \\ y_{1} y_{2}-z_{1} z_{2}, & \text { if } x_{1}=0 \wedge x_{2}=0\end{cases}
$$

where $v_{1}=\left(x_{1}, y_{1}, z_{1}\right)$ and $v_{2}=\left(x_{2}, y_{2}, z_{2}\right)[6]$.

A vector $v=(x, y, z)$ is said to be non-isotropic if $x \neq 0$. All unit non-isotropic vectors are the form $(1, y, z)$. For isotropic vectors $x=0$ holds. There are four types of isotropic vectors: spacelike $\left(y^{2}-z^{2}>0\right)$, timelike $\left(y^{2}-z^{2}<0\right)$ and two types of lightlike $(y= \pm z)$ vectors. A non-lightlike isotropic vector is a unit vector if $y^{2}-z^{2}= \pm 1$.

The pseudo-Galilean cross product $\times$ of $v_{1}$ and $v_{2}$ on $G_{3}^{1}$ is defined by

$$
v_{1} \times v_{2}=\left|\begin{array}{ccc}
0 & -e_{2} & e_{3} \\
x_{1} & y_{1} & z_{1} \\
x_{2} & y_{2} & z_{2}
\end{array}\right|,
$$

where $e_{2}=(0,1,0)$ and $e_{3}=(0,0,1)$ (for detail, see [6], [7], [15] and etc.).

In a tangent plane of a surface parametrized by

$$
\Gamma(u, v)=(x(u, v), y(u, v), z(u, v))
$$

in a point $P_{0}$, there is a unique isotropic direction defined by the condition $x_{u} d u+x_{v} d v=0$, where $x_{u}=\frac{\partial x}{\partial u}, x_{v}=\frac{\partial x}{\partial v}$. A side tangential vector $\sigma=\frac{1}{W}\left(x_{u} \Gamma_{v}-x_{v} \Gamma_{u}\right)$ is a unit isotropic vector in a tangent plane. The function $W>0$, defined by

$$
W=\sqrt{\left|\left(x_{u} y_{v}-x_{v} y_{u}\right)^{2}-\left(x_{u} z_{v}-x_{v} z_{u}\right)^{2}\right|}
$$

is equal to the pseudo-Galilean norm of the side tangential vector $\sigma$. Also, a unit surface normal field is defined by

$$
N=\frac{1}{W}\left(0, x_{u} z_{v}-x_{v} z_{u}, x_{u} y_{v}-x_{v} y_{u}\right)
$$

Since $\langle N, N\rangle= \pm 1=\varepsilon$, we distinguish between two types of admissible surfaces: spacelike surfaces having timelike surface normals and timelike surfaces having spacelike normals. 
The second fundamental form $I I$ is given by

$$
I I=L_{11} d u^{2}+2 L_{12} d u d v+L_{22} d v^{2},
$$

where $L_{i, j}, i, j=1,2$, are the normal components of $\Gamma_{u u}, \Gamma_{u v}, \Gamma_{v v}$, respectively and it holds

$$
L_{i j}=\varepsilon<\frac{1}{x_{u}}\left(x_{u} \tilde{\Gamma}_{i j}-x_{i j} \tilde{\Gamma}_{u}\right), \tilde{N}>=\varepsilon<\frac{1}{x_{v}}\left(x_{v} \tilde{\Gamma}_{i j}-x_{i j} \tilde{\Gamma}_{v}\right), \tilde{N}>.
$$

By $\sim$ above, the projection of a vector on the pseudo-Euclidean $y z$-plane is denoted.

The Gaussian and mean curvatures of a surface defined by means of the coefficients of the second fundamental form are defined by

$$
K=-\varepsilon \frac{L_{11} L_{22}-L_{12}^{2}}{W^{2}}
$$

and

$$
H=-\varepsilon \frac{x_{v}^{2} L_{11}-2 x_{u} x_{v} L_{12}^{2}+x_{u}^{2} L_{22}}{2 W^{2}},
$$

respectively [7].

Furthermore, a twisted surface in $E^{3}$ or $E_{1}^{3}$ is obtained by rotating a planar curve $\alpha$ in its supporting plane while this plane itself is rotated about some containing straight line [8].

\section{Twisted surfaces in $G_{3}^{1}$}

With the same construction of a twisted surface in Euclidean 3-space $E^{3}$ and Minkowski 3-space $E_{1}^{3}$, the planar curve $\alpha$ rotates in its supporting plane while this plane itself rotates about some containing straight line in the pseudo-Galilean space $G_{3}^{1}$.

Since a straight line can be either spacelike, timelike or lightlike in $G_{3}^{1}$ we have to consider several possibilities. Therefore, we need to know how a rotation about an axis in $G_{3}^{1}$ is described.

In the pseudo-Galilean space $G_{3}^{1}$, there are two types of rotations.

Pseudo-Euclidean rotations given by the normal form

$$
\begin{aligned}
& \bar{x}=x, \\
& \bar{y}=y \cosh t+z \sinh t, \\
& \bar{z}=y \sinh t+z \cosh t
\end{aligned}
$$

and isotropic rotations with the normal form

$$
\begin{aligned}
& \bar{x}(t)=x+b t, \\
& \bar{y}(t)=y+x t+b \frac{t^{2}}{2}, \\
& \bar{z}(t)=z,
\end{aligned}
$$


where $t \in \mathbb{R}$ and $b=$ const $>0[14]$.

In the pseudo-Galilean space $G_{3}^{1}$, the $x$-axis and the $y$-axis are spacelike while the $z$-axis is timelike. So, the $x y$-plane is spacelike while $x z$-plane and the $y z$-plane are timelike. Here, we will construct the twisted surfaces according to the supporting plane and type of rotations in pseudo-Galilean space.

\section{(1) Let the profile curve lies in a timelike plane.}

Let we assume that the profile curve $\alpha$ lies in the $y z$-plane. So, it can be parametrized as $\alpha(u)=(0, f(u), g(u))$.

\section{(a) A pseudo-Euclidean rotation and an isotropic rotation.}

If we rotate $\alpha$ about the straight line through the point $(0, a, 0)$ parallel with the $x$-axis, then we obtain

$$
\left[\begin{array}{l}
0 \\
a \\
0
\end{array}\right]+\left[\begin{array}{ccc}
1 & 0 & 0 \\
0 \cosh (c v) & \sinh (c v) \\
0 & \sinh (c v) & \cosh (c v)
\end{array}\right]\left[\begin{array}{c}
0 \\
f(u) \\
g(u)
\end{array}\right]=\left[\begin{array}{c}
0 \\
a+f(u) \cosh (c v)+g(u) \sinh (c v) \\
f(u) \sinh (c v)+g(u) \cosh (c v)
\end{array}\right]
$$

Now, applying an isotropic rotation to (14), i.e. by rotating (14) about the $z$-axis, we have the following twisted surface in $G_{3}^{1}$.

$$
\left[\begin{array}{lll}
1 & 0 & 0 \\
v & 1 & 0 \\
0 & 0 & 1
\end{array}\right]\left[\begin{array}{c}
0 \\
a+f(u) \cosh (c v)+g(u) \sinh (c v) \\
f(u) \sinh (c v)+g(u) \cosh (c v)
\end{array}\right]+b\left[\begin{array}{c}
v \\
\frac{v^{2}}{2} \\
0
\end{array}\right]=\left[\begin{array}{c}
b v \\
a+f(u) \cosh (c v)+g(u) \sinh (c v)+b \frac{v^{2}}{2} \\
f(u) \sinh (c v)+g(u) \cosh (c v)
\end{array}\right], a, b, c \in \mathbb{R}
$$

So, a parametrization of the twisted surface in this situation is

$$
\Gamma(u, v)=\left(b v, a+f(u) \cosh (c v)+g(u) \sinh (c v)+b \frac{v^{2}}{2}, f(u) \sinh (c v)+g(u) \cosh (c v)\right) .
$$

(Here, the presence of the factor $c \in \mathbb{R}$ allows for differences in the rotation speed of both rotations.)

Figure 1 shows the twisted surface (15) with profile curve the Lemniscates of Bernoulli, $\alpha(u)=\left(0, \frac{a_{1} \cos u}{1+\sin ^{2} u}, \frac{a_{1} \sin u \cos u}{1+\sin ^{2} u}\right)$, for constants $a_{1}=4, a=3, b=2, c=1$.

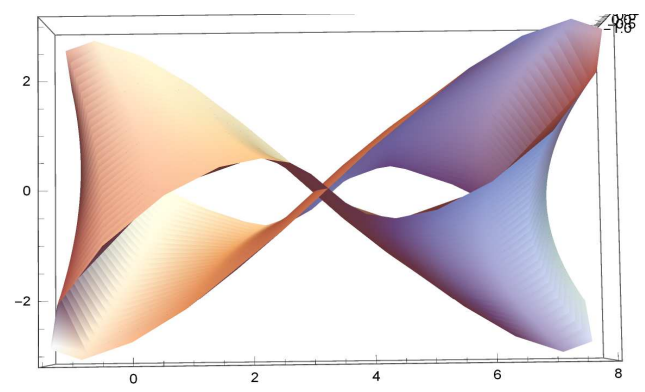

Fig. 1

Here, let us find the Gaussian and mean curvature of the twisted surface (15). 
Let us assume that the profile curve $\alpha$ is parametrized by the arc length, i.e.

$$
f^{\prime 2}(u)-g^{\prime 2}(u)=-\varepsilon, \varepsilon= \pm 1 .
$$

From (6), we have

$$
W=b,
$$

and from (7) and (16), the normal field of the twisted surface (15) is

$$
N=-\left(0, f^{\prime}(u) \sinh (c v)+g^{\prime}(u) \cosh (c v), f^{\prime}(u) \cosh (c v)+g^{\prime}(u) \sinh (c v)\right) .
$$

Here, one can easily see that $\langle N, N\rangle=\varepsilon$. Furthermore, from (9), (15) and (18), the components of the second fundamental form can be found as

$$
\begin{aligned}
& L_{11}=-\varepsilon\left\{f^{\prime \prime} g^{\prime}-f^{\prime} g^{\prime \prime}\right\}, \\
& L_{12}=-c, \\
& L_{22}=-\varepsilon\left\{c^{2} f g^{\prime}-c^{2} f^{\prime} g+b f^{\prime} \sinh (c v)+b g^{\prime} \cosh (c v)\right\} .
\end{aligned}
$$

So, we can state the following Theorem.

Theorem 1. The Gaussian and mean curvatures of the twisted surface (15) are

$$
\begin{aligned}
K=- & \varepsilon\left\{\frac{c^{2}}{b^{2}}\left\{f f^{\prime \prime} g^{2}-f^{\prime} f^{\prime \prime} g g^{\prime}-f f^{\prime} g^{\prime} g^{\prime \prime}+f^{\prime 2} g g^{\prime \prime}-1\right\}\right. \\
& \left.+\frac{1}{b}\left\{\left(f^{\prime} f^{\prime \prime} g^{\prime}-f^{\prime 2} g^{\prime \prime}\right) \sinh (c v)+\left(f^{\prime \prime} g^{\prime 2}-f^{\prime} g^{\prime} g^{\prime \prime}\right) \cosh (c v)\right\}\right\}
\end{aligned}
$$

and

$$
H=\frac{1}{2}\left\{f^{\prime \prime} g^{\prime}-f^{\prime} g^{\prime \prime}\right\}
$$

respectively.

Proof. From (10), (11) and (19), we obtain (20) and (21).

Corollary 1. If the twisted surface (15) is minimal, then we get

$$
g(u)=c_{1} u+c_{2} \text { and } f(u)=\sqrt{c_{1}^{2}-\varepsilon} \cdot u+c_{3} ; c_{1}, c_{2}, c_{3} \in \mathbb{R}
$$

and so, the minimal twisted surface (15) can be parametrized as

$$
\begin{aligned}
\Gamma(u, v)= & \left(b v, a+\left(\sqrt{c_{1}^{2}-\varepsilon} \cdot u+c_{3}\right) \cosh (c v)+\left(c_{1} u+c_{2}\right) \sinh (c v)+b \frac{v^{2}}{2},\right. \\
& \left.\left(\sqrt{c_{1}^{2}-\varepsilon} \cdot u+c_{3}\right) \sinh (c v)+\left(c_{1} u+c_{2}\right) \cosh (c v)\right) .
\end{aligned}
$$

Proof. The proof is obvious from (16) and (21).

\section{(b) Twice pseudo-Euclidean rotations.}

If we rotate $\alpha$ about the straight line through the point $(0,0, a)$ parallel with the $x$-axis and again applying a pseudoEuclidean rotation to this surface, i.e. by rotating this surface about the $y$-axis, we have the following twisted surface in $G_{3}^{1}$.

$$
\Gamma(u, v)=(a+f(u) \sinh (c v)+g(u) \cosh (c v)) \cdot(\sinh v, 0, \cosh v)+(0, f(u) \cosh (c v)+g(u) \sinh (c v), 0) .
$$

Figure 2 shows the twisted surface (23) with profile curve $\alpha(u)=(0, \cos u, \sin u)$ for constants $a=2, c=3$. 


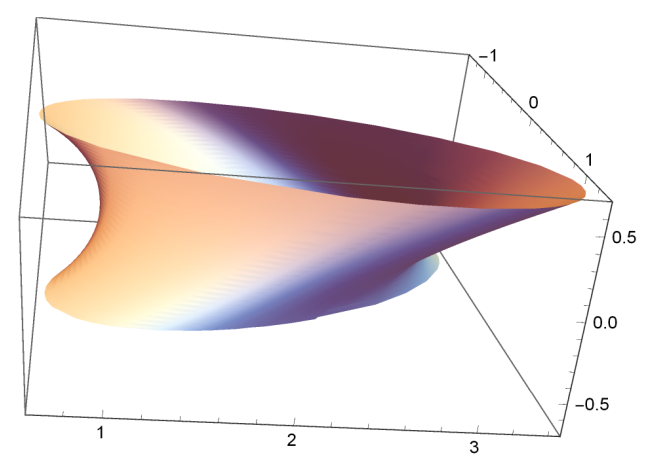

Fig. 2

The Gaussian and mean curvature of the twisted surface (23) can be found as the above procedure after long calculations.

\section{(2) Let the profile curve lies in a spacelike plane.}

Let we assume that the profile curve $\alpha$ lies in the $x y$-plane. So, it can be parametrized as $\alpha(u)=(f(u), g(u), 0)$.

Here, if we apply an isotropic rotation to $\alpha$ about the straight line through the point $(0, a, 0)$ parallel with the $z$-axis followed by a pseudo-Euclidean rotation about $x$-axis, then the obtain the following twisted surface in $G_{3}^{1}$.

$$
\Gamma(u, v)=\left(a+c f(u) v+g(u)+b \frac{c^{2} v^{2}}{2}\right) \cdot(0, \cosh v, \sinh v)+(f(u)+b c v, 0,0) .
$$

If we assume that the profile curve is parametrized by arc length, then the twisted surface (24) can be parametrized as

$$
\Gamma(u, v)=\left(u+b c v,\left(a+c u v+g(u)+b \frac{c^{2} v^{2}}{2}\right) \cdot \cosh v,\left(a+c u v+g(u)+b \frac{c^{2} v^{2}}{2}\right) \cdot \sinh v\right) .
$$

Therefore,

Theorem 2. The Gaussian and mean curvature of the twisted surface (25) are

$$
K=\frac{D^{2}-C^{2}}{W^{6}}\left\{g^{\prime \prime} C \cdot\left(b c^{2} C-2 B D+C^{2}\right)-(c C-A D)^{2}\right\}
$$

and

$$
H=-\frac{\left(D^{2}-C^{2}\right)^{2}}{2 W^{7}}\left\{b^{2} c^{2} C g^{\prime \prime}-b c^{2} C+2 b c A D-2 B D+C^{2}\right\}
$$

respectively, where

$$
A=c v+g^{\prime}, B=c u+b c^{2} v, C=a+c u v+g+b c^{2} \frac{v^{2}}{2}, D=c u-b c g^{\prime}, W=\sqrt{\left|D^{2}-C^{2}\right|} .
$$

Proof. The proof is obvious from (9)-(11).

Figure 3 shows the twisted surface (24) with profile curve $\alpha(u)=(\sin u+5, \cos u-2,0)$ for constants $a=4, b=2, c=3$. 


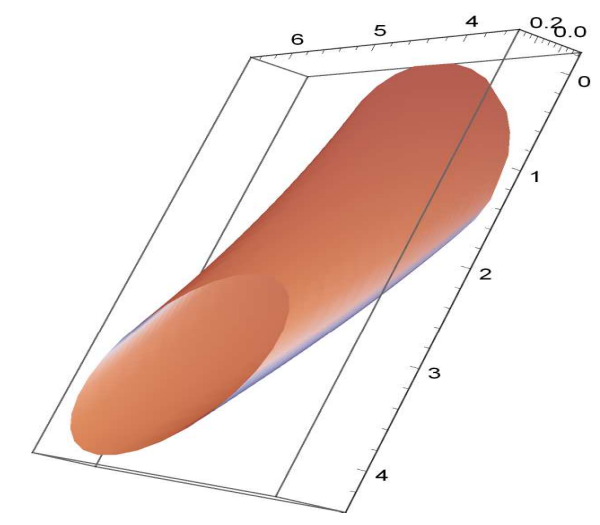

Fig. 3

\section{Competing interests}

The authors declare that they have no competing interests.

\section{Authors' contributions}

All authors have contributed to all parts of the article. All authors read and approved the final manuscript.

\section{References}

[1] C. Baikoussis, D.E. Blair; On the Gauss map of ruled surfaces, Glasgow Math. J. 34, 355-359, 1992.

[2] S. Cengiz, E.B. Koç Öztürk and U. Öztürk; Motions of Curves in the Pseudo-Galilean Space $G_{3}^{1}$, Mathematical Problems in Engineering, http://dx.doi.org/10.1155/2015/150685, 2015.

[3] D. Cervone; A tight polyhedral immersion of the twisted surface of Euler characteristic -3, Topology 40, 571 - 584, 2001.

[4] B.Y. Chen, M. Choi, Y.H. Kim; Surfaces of revolution with pointwise 1-type Gauss map, J. Korean Math. Soc. 42(3), 447-455, 2005.

[5] B.Y. Chen, S. Ishikawa; On classification of some surfaces of revolution of finite type, Tsukuba J. Math. 17, 287-298, 1993.

[6] B. Divjak; Curves in Pseudo-Galilean geometry, Ann. Univ. Sci. Budapest. Eötvös Sect. Math. 41, 117-128, 1998.

[7] B. Divjak and Z. M. Sipus; Some special surfaces in the pseudo-Galilean space, Acta Math. Hungar. 118, 209-226, 2008.

[8] W. Goemans and I.V. de Woestyne; Twisted surfaces in Euclidean and Minkowski 3-space, Pure and Applied Differential Geometry, 143-151, 2013.

[9] W. Goemans and I.V. de Woestyne; Twisted Surfaces with Null Rotation Axis in Minkowski 3-Space, Results. Math., DOI 10.1007/s00025-015-0462-2, 2015.

[10] W. Goemans and I.V. de Woestyne; Constant curvature twisted surfaces in 3-dimensional Euclidean and Minkowski space. In: Proceedings of the Conference RIGA 2014. Riemannian Geometry and Applications to Engineering and Economics, pp. 117-130. Bucharest, 2014.

[11] A. Kazan, H.B. Karadağ; A Classification of Surfaces of Revolution in Lorentz-Minkowski Space, Int. J. Contemp. Math. Sciences 6(39), 1915-1928, 2011.

[12] A. Kazan, H.B. Karadağ; Surfaces of Revolution in Minkowski 3-Space Satisfying $\tilde{\Gamma}_{11}^{1}(G)=k(G+C)$, J. of Math. and System Sci. 3, 567-572, 2013.

[13] Y.H. Kim; Ruled surfaces and their Gauss maps in Lorentz-Minkowski spaces, Information Center for Mathematical Sciences 5(2), 97-104, 2002. 
[14] Z.M. Sipus and B. Divjak; Surfaces of Constant Curvature in the Pseudo-Galilean Space, Int. J. of Math. and Math. Sci., doi:10.1155/2012/375264, 2012.

[15] D.W. Yoon; Surfaces of Revolution in the Three Dimensional Pseudo-Galilean Space, Glasnik Matematicki 48(68), 415 - 428 , 2013.

[16] D.W. Yoon; On the Gauss map of Tubular Surfaces in Galilean 3-space, Int. J. of Math. Analysis 8(45), 2229-2238, 2014.

[17] D.W. Yoon; Some Classification of Translation Surfaces in Galilean 3-Space, Int. J. of Math. Analysis 6(28), 1355-1361, 2012. 